\title{
IT-Kernkompetenzen im Bachelorstudiengang „Informationswissenschaften“ an der Hochschule der Medien Stuttgart
}

https://doi.org/10.1515/bfp-2019-2052

Zusammenfassung: Die Lehrangebote im Bereich der informationstechnischen Grundlagen im Studiengang „Informationswissenschaften“ an der HdM Stuttgart wurden in den vergangenen Jahren grundlegend überarbeitet und völlig neu strukturiert. Sie werden nun im „flipped classroom" Modell unterrichtet und adressieren neben fachlichen Inhalten wie Programmierung, Datenstrukturen und -verarbeitung sowie webbasierte Angebote auch nichtfachliche Kompetenzen wie Frustrationstoleranz im Umgang mit IT, eigenständiges Arbeiten und Lernen sowie allgemeine Problemlösungsstrategien. Das Konzept konnte erfolgreich im Lehrbetrieb umgesetzt werden und wird im neu angebotenen Schwerpunkt „Daten- und Informationsmanagement“ des Studiengangs weiter ausgebaut.

Schlüsselwörter: Informationstechnische Kompetenz; informationswissenschaftliches Studium; bibliothekswissenschaftliches Studium; didaktisches Konzept

\section{Core IT Competencies in the Undergraduate Course "Infor- mation Sciences" at Stuttgart Media University}

\begin{abstract}
Over the last few years, the School of Information Sciences at Stuttgart Media University has profoundly changed those parts of the curriculum concerned with information technology competencies and created a completely restructured set of modules. These modules are being taught in a flipped classroom environment and cover not only topics like programming, data structures, data processing or web-based systems but also key competencies like how to develop frustration tolerance when working with IT, methods for independent learning and working as well as general problem solving strategies. This new curriculum has been successfully implemented and will be further refined in the new focus area "Data and Information Management”.
\end{abstract}

*Kontaktperson: Magnus Pfeffer, pfeffer@hdm-stuttgart.de
Keywords: Information technology competency; information science curriculum; library science curriculum; teaching concept

\section{Einleitung}

In den vergangenen fünf Jahren gab es zwei umfassende Überarbeitungen der Studienordnung des Studiengangs „Bibliotheks- und Informationsmanagement“ bzw. „Informationswissenschaften "1 der Hochschule der Medien Stuttgart. Anlässe und Schwerpunkte der Anpassungen waren unterschiedlich, aber in beiden Fällen spielten Kompetenzen und Inhalte im Bereich der Informationstechnologie eine wesentliche Rolle.

Dieser Beitrag fasst den Ablauf und die Ergebnisse des Diskussions- und Umsetzungsprozesses zusammen und zeigt auf, wie sich der Studiengang zukünftig positioniert. Zunächst werden die Änderungsprozesse in ihrem weiteren Kontext beschrieben und die wesentlichen Anpassungen im Bereich IT erläutert. Anschließend werden Konzept, Planung, Inhalte und didaktische Umsetzung der Grundlagenmodule vorgestellt. Es folgt eine Auswertung der Erfahrungen nach fünf Semestern Einsatz in der Praxis und deren Einfluss auf die aktuelle Studienordnung, die zum Wintersemester 2018/19 in Kraft getreten ist. Ein Überblick über diese Studienordnung und den neu eingeführten Schwerpunkt „Daten- und Informationsmanagement“ schließt den Bericht ab.

\section{Veränderungsprozesse}

Inhaltliche Veränderungen eines Studienangebots sind oftmals in größere Veränderungsprozesse eingebettet, die sich auf die Rahmenbedingungen auswirken oder eine strategische Ausrichtung vorgeben. Die wesentlichen Vor-

1 Der Studiengang trägt ab dem Sommersemester 2019 den Namen „Informationswissenschaften“. Zuvor hieß er „Bibliotheks- und Informationsmanagement“. 
gänge der letzten Jahre sollen hier kurz zusammengefasst werden.

Die Umstellung der traditionellen Diplomstudiengänge auf den Bachelor-Abschluss im Rahmen des BolognaProzesses und die damit einhergehende Modularisierung der Studieninhalte wurde an der Hochschule der Medien in Stuttgart frühzeitig angegangen. Bereits zum Wintersemester 2004/05 war dieser Vorgang für alle Studiengänge abgeschlossen, wobei zunächst das Modell „6+4“, also ein sechssemestriges Bachelorstudium mit der Möglichkeit eines konsekutiven, viersemestrigen Masterstudiums, genutzt wurde. ${ }^{2}$ Nach einer Auswertung der Erfahrungen der ersten Jahrgänge wurde von den Gremien der Hochschule der Umstieg auf das Modell „7+3“ beschlossen. Die Anzahl der Prüfungsleistungen pro Semester sollte reduziert, die Inhalte sollten entzerrt und den Studierenden mehr Möglichkeiten zum Setzen eigener Schwerpunkte im Hauptstudium gegeben werden. Der Studiengang setzte diese Vorgabe zum Wintersemester 2011/12 um.

Diese Studienordnung beinhaltete im Bereich IT zwei Module im Grundstudium, die neben allgemeinem Hintergrundwissen zu Hardware, Betriebssystemen, Anwendungen und Computernetzen vor allem den relationalen Datenbankentwurf und die Nutzung von SQL in den Mittelpunkt stellten. Im Hauptstudium folgte das Modul „IT-Management“, in dem Bibliotheksinformationssysteme, IT-Management mit der IT Infrastructure Library (ITIL) sowie technische und organisatorische Grundlagen digitaler Bibliotheken Thema waren. Während die Module des Grundstudiums neben einer Vorlesung auch praktische Übungen umfassten, wurden die Inhalte des Hauptstudiums in drei Vorlesungen vermittelt.

Innerhalb der Fakultät Information und Kommunikation, der der Studiengang angehört, begann 2011 ein längerer Diskussionsprozess, in dem die zukünftige Ausrichtung der Fakultät, die Inhalte und Struktur der Studiengänge bis hin zu unterschiedlichen didaktischen Formaten hinterfragt und über die richtige Vorgehensweise teils auch kontrovers debattiert wurde. Dieser Prozess wurde flankiert von einem intensiven Erfahrungsaustausch mit KollegInnen aus anderen Hochschulen und in mehreren Workshops, teilweise mit externer Moderation, unter Beteiligung aller Mitglieder der Fakultät und Studierendenvertretern durchgeführt. ${ }^{3}$ Die Ausgangslage stellte sich wie folgt dar:

$2 \mathrm{Zu}$ den konkreten Inhalten und dem Aufbau des sechssemestrigen Bachelorstudiengangs „Bibliotheks- und Informationsmanagement“ siehe Simon und Vonhof (2008).

3 Eine wesentlich ausführlichere Darstellung dieses Prozesses findet sich in Vonhof (2017).
- Die dynamische gesellschaftliche und technische Entwicklung verändert in vielen Bereichen die Lebensund Arbeitswelten massiv und wird es auch in Zukunft tun. Die Studierenden der Fakultät sollen auf eine Welt vorbereitet werden, in der etabliertes Wissen schnell veralten kann und es erforderlich ist, sich immer wieder auf neue Anforderungen einzustellen und sich eigenständig neues Wissen oder neue Arbeitstechniken anzueignen.

- Die Hochschulen für Angewandte Wissenschaften in Baden-Württemberg werden von zwei Seiten bedrängt: Die Universitäten sind stark auf Forschung fokussiert und punkten mit einer deutlich besseren technischen und personellen Ausstattung, während die Duale Hochschule ein Studium mit einer starken Verzahnung mit der beruflichen Praxis anbietet. Um in diesem Wettbewerb bestehen zu können und von den Studierenden als attraktive Alternative wahrgenommen zu werden, reicht ein „weiter so“ mit den bestehenden Konzepten nicht aus.

- Interdisziplinarität und projektbezogenes Arbeiten bestimmt in zunehmendem Maße den Arbeitsalltag. Komplexe Fragestellungen werden in gemischten Teams bearbeitet, deren Mitglieder nicht nur die eigene fachliche Sicht einbringen, sondern die unterschiedlichen Arbeits- und Herangehensweisen der anderen verstehen und mit diesen effektiv zusammenarbeiten müssen. Ein Studium, das sich allein auf seine fachlichen Inhalte fokussiert, kann auf eine solche Arbeitswelt nur unzureichend vorbereiten.

Im Ergebnis wurde ein für alle Studiengänge der Fakultät einheitliches Strukturmodell entwickelt, das optimale Rahmenbedingungen bietet, um den zentralen Herausforderungen zu begegnen. Dabei wird die traditionelle fachliche Ausbildung, die in den ersten vier Semestern erfolgt, von fakultätsweiten Schlüsselqualifikationsmodulen ergänzt, die wichtige Soft Skills vermitteln. Zentral ist außerdem ein Modul „Wissenschaftliche Grundlagen“, das über zwei Semester einen Überblick über den vollständigen wissenschaftlichen Arbeitsprozess gibt und gezielt in den zwischen den Studiengängen durchmischten Gruppen durchgeführt wird. Insgesamt werden die Schlüsselqualifikationen inkl. dem wissenschaftlichen Arbeiten stark aufgewertet und machen 25 der 120 ECTS der ersten vier Semester aus. Nach dem praktischen Studiensemester sind im 6. und 7. Semester neben zwei kleineren Modulen zur freien fachlichen Spezialisierung nur noch projektbasierte Lehr- und Lernformen vorgesehen. Die Palette umfasst dabei drei Typen: erstens kleinere, fachlich fokussierte Projekte im Umfang von 5 ECTS; zweitens interdisziplinäre 
Projekte im Umfang von 10 ECTS, in denen mindestens zwei unterschiedliche fachliche Sichten in die Fragestellung eingehen und von unterschiedlichen DozentInnen vertreten werden. In der Regel sind in interdisziplinäre Projekte externe Partner integriert. Als Rahmen für besonders herausfordernde Projekte sind, drittens, transdisziplinäre Projekte vorgesehen, die sich über zwei Semester erstrecken und insgesamt 20 ECTS umfassen. Jeder Studierende muss mindestens ein interdisziplinäres oder transdisziplinäres Projekt im Laufe des Studiums absolvieren.

Alle Studiengänge der Fakultät passten ihre Studienordnungen an dieses Modell an und zum Wintersemester 2016/17 startete der erste Jahrgang mit dem neuen Konzept. Der Studiengang Bibliotheks- und Informationsmanagement wählte eine Säulenstruktur, die die Inhalte des Grund- und Hauptstudiums ordnet: Bibliothek \& Informationsorganisation, Informationssysteme, Kultur \& Bildung und Public Management. Um trotz der neu hinzugekommenen Schlüsselqualifikationsmodule und dem Projektstudium noch einen Freiraum für individuelle Spezialisierung zu schaffen, wurden dabei die Inhalte der vorherigen Studienordnung kritisch hinterfragt und von den fachlichen Vertretern der einzelnen Säulen in ein reduziertes Pflichtprogramm mit passenden Vertiefungen in Wahlangeboten aufgeteilt. Die Studierenden müssen dabei neben den im Strukturmodell vorgesehenen beiden Modulen im 6. und 7. Semester bereits im 3. und 4. Semester sechs weitere Wahlmodule belegen und können so die fachliche Spezialisierung ihres Studiums gestalten. Insbesondere im Bereich der Informationssysteme wurde mit den Inhalten der vorherigen Studienordnung gebrochen und ein komplett neues Programm entworfen, das im Folgenden genauer vorgestellt wird.

\section{Module der Säule „Informationssysteme“}

Das hier vorgestellte Modell einer IT-Grundausbildung im Rahmen eines bibliothekarischen Studiengangs greift die Ausgangslage der fakultätsweiten Diskussion auf und versucht, für die fachliche Säule die Frage zu beantworten, welche Kompetenzen geeignet sind, die Studierenden sowohl für die Mitarbeit in interdisziplinären Projekten als auch für eine Arbeitswelt vorzubereiten, die durch die fortschreitende Digitalisierung einem steten und teilweise sehr starkem Wandel unterliegt. Dabei wurden folgende Grundannahmen getroffen:

- Konkrete Kenntnisse in der Anwendung spezifischer Anwendungssoftware und Betriebssystemen oder be- stimmter Hardware sind nur schwer zu verallgemeinern oder auf andere Fragestellungen anwendbar. Darüber hinaus sind sie durch den technischen Fortschritt bereits nach kurzer Zeit obsolet.

- Passive Kenntnisse der Grundlagen sind im IT-Bereich nicht ausreichend. Es ist erforderlich, eigene Erfahrungen in der Anwendung und Bearbeitung von Aufgaben zu machen, um diese in Projekten bei der Lösung konkreter Probleme einsetzen zu können.

- Studierende, die sich für das Fach entscheiden, bringen nicht unbedingt eine große Neigung zur Beschäftigung mit Fragestellungen aus dem Bereich der Informationstechnik mit. Es ist deshalb erforderlich, kontinuierlich die Relevanz der Kenntnisse zu vermitteln und durch die didaktische Aufbereitung der Inhalte auch denjenigen, die mit IT „fremdeln“, einen Zugang zu dem Themenbereich zu schaffen.

In der Diskussion der fachlichen Vertreter wurde schnell klar, dass gleichberechtigt neben der Vermittlung fachlicher Inhalte wichtige Kernkompetenzen vermittelt werden müssen, die für die Arbeit mit (informations)technischen Systemen erforderlich sind:

\section{- Aufbau von Frustrationstoleranz}

Die Einarbeitung in unbekannte Softwareanwendungen oder gar die Umsetzung eigener Ideen mittels Software ist stets mit einer vergleichsweise steilen Lernkurve verbunden: Anleitungen können unvollständig, unverständlich oder nicht vorhanden sein. Menü-Einträge und Bezeichnungen sind mitunter kryptisch, Fehlermeldungen wenig hilfreich. Fehlersuche in fremden oder eigenen Programmen kann sich über lange Zeiträume hinziehen. Es ist daher zentral, die Studierenden auf solche Situationen vorzubereiten und ihnen Strategien zu vermitteln, damit umzugehen.

- Eigenständiges Erarbeiten von Inhalten

Aufgrund des schnellen technischen Wandels müssen die Studierenden von Anfang an verstehen, dass es nicht so sein wird, dass die Lehrenden die Inhalte für sie häppchenweise erläutern und sie diese dann lernen und damit „fertig“ sind. Das eigene Lernverhalten zu reflektieren und in der Lage zu sein, für neue Fragestellungen geeignete Lernmaterialien selbst zu finden, wird für die Studierenden wichtig sein.

- Allgemeine Problemlösekompetenz.

Komplexe und schwierige Probleme und Fragestellungen in kleinere, handhabbare und leichter verständliche Teilprobleme zu zerlegen und deren Teillösungen anschließend zu kombinieren, ist eine Kernstrategie in der Anwendung von Informationstechnik - egal ob es 
sich dabei um die Einführung einer neuen Anwendungssoftware oder das Erstellen eines eigenen Programms handelt. Die Studierenden müssen dies in möglichst vielen Kontexten immer wieder üben und Erfahrungen damit sammeln.

Auf der fachlichen Seite wurden Inhalte ausgewählt, die auch bei einer schnellen technischen Entwicklung zentral sind und eine solide Grundlage für darauf aufbauende, komplexere Vertiefungsangebote bilden:

- Grundlegende Programmierkenntnisse

Variablen und Operatoren, lineare und verzweigte Ausführung, Schleifen, Funktionen und Datenstrukturen wie Listen oder assoziative Arrays sind die wesentlichen Elemente aller Programmiersprachen. Studierende sollen in der Lage sein, Aufgabenstellungen in die formale Struktur von Programmen zu übertragen und eigene Skripte und Programme zu erstellen.

\section{- Arbeiten mit Daten}

Dies umfasst die Auswahl geeigneter Datenstrukturen und Datenformate für Speicherung, Aufbau und Strukturierung von Datenbanken und die Verarbeitung von Daten, z.B. Filterung, Suche und Kombination von Daten aus mehreren Quellen.

\section{- Web-basierte Anwendungen}

Viele Anwendungen werden inzwischen als Web-Anwendung bereitgestellt und dieser Trend zur Softwareas-a-service wird weiter anhalten. Entsprechend sollten die Studierenden die Grundlagen des Webs, also das http-Protokoll und die Funktion von Webservern, kennen und eigene Webanwendungen durch Kombination von HTML, CSS und eigenen Programmen erstellen können.

Konkrete Anwendungen sind somit nicht Inhalt der Module der Säule Informationssysteme; sie werden stattdessen in den anderen fachlichen Vorlesungen im jeweiligen Kontext besprochen. Bibliotheksinformationssysteme bilden Geschäftsprozesse in Bibliotheken ab und sind bei der Vorstellung der Geschäftsgänge ein Thema; Authentifizierungsverfahren sind für die Umsetzung der Zugangskontrolle von lizenzierten digitalen Medien erforderlich und gehören somit zum Kontext von Erwerbung bzw. Lizenzierung. Kataloge und Suchmaschinen sind Informationsressourcen und ihre Funktionen und Dienste wie z. B. Link Resolver sollen in diesem Kontext erläutert werden.

Insgesamt werden drei grundlegende Module in der Säule Informationssysteme angeboten, die jeweils 5 ECTS umfassen und aufeinander aufbauen. Allen gemein ist das didaktische Konzept des „flipped classroom“: Neue Inhalte werden von den Studierenden eigenständig zuhau- se erarbeitet. Dazu stehen Lernmaterialien in der E-Learning-Plattform bereit; Übungsaufgaben, die in kleinen Gruppen bearbeitet werden, fordern das neu Gelernte direkt ein. Die Kontaktzeit in der Vorlesung kann für die Diskussion der Übungsaufgaben, Erläuterungen bei Verständnisschwierigkeiten oder für individuelle Unterstützung genutzt werden. Die Inhalte und didaktische Aufbereitung der einzelnen Module werden im Folgenden kurz vorgestellt.

\subsection{Informationssysteme 1: Programmieren}

Um einen niedrigschwelligen Einstieg anzubieten, der keine Vorkenntnisse erfordert und somit auch Studierenden, die der Thematik eher skeptisch gegenüberstehen, einen Zugang bieten kann, wurde für die Einführung gezielt eine Programmierumgebung ausgewählt, die intuitiv zu erlernen ist und anstelle komplizierter Syntax graphische Repräsentationen der Programmbausteine einsetzt. Snap! ${ }^{4}$ ist eine visuelle Programmiersprache, in der Programme aus solchen Bausteinen zusammengeklickt werden. Sie wird von der University of California in Berkeley als Web-Anwendung bereitgestellt; zur Nutzung wird nur ein aktueller Browser wie Chrome oder Mozilla Firefox benötigt.

Abb. 1 zeigt einen Ausschnitt aus einem Snap!-Programm. Snap! umfasst alle wesentlichen Elemente einer Programmiersprache und unterstützt audiovisuelle Elemente wie bewegte Sprites, Hintergründe, Töne oder Hinweistexte in Sprechblasen. Studierende können so sehr schnell erste Erfolgserlebnisse mit einfachen eigenen Programmen erleben, aber auch komplexere Probleme bearbeiten. Im Modul wird parallel zur Einführung der Grundlagen den Studierenden ein Überblick über verschiedene Lehrmedien zu Snap! gegeben, die die Studierenden ausprobieren und somit ein erstes Gefühl für den eigenen Lerntyp und bevorzugte Lernmaterialen entwickeln. Die erste Phase des Moduls endet in einer Gruppenarbeit, in der ein interaktives Spiel konzeptioniert, programmiert und mit eigener Grafik gestaltet wird. Abb. 2 zeigt einen Screenshot einer studentischen Arbeit aus dieser Phase.

Mit zunehmender Komplexität der eigenen Programme wird den Studierenden auch die Schwäche einer visuellen Programmiersprache bewusst: Große Programme werden schnell unübersichtlich, die Bearbeitung ausschließlich mit der Maus ist ermüdend und die Grenzen der Programmierumgebung werden bei der Programmierung der Spiele sichtbar.

4 https://snap.berkeley.edu. 


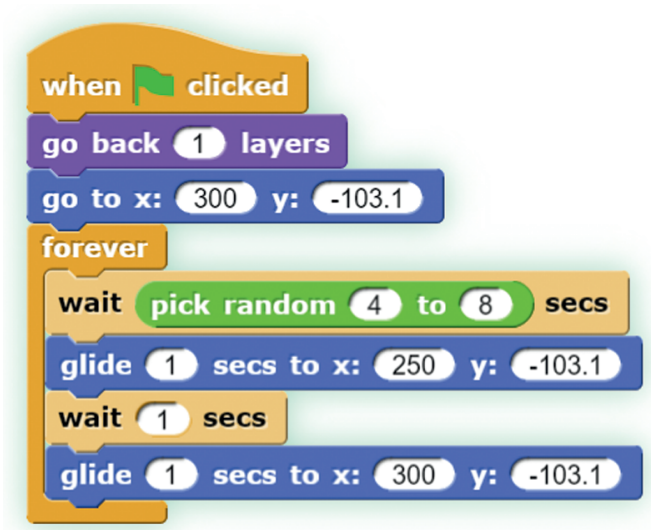

Abb. 1: Beispielprogramme der visuellen Programmiersprache Snap!

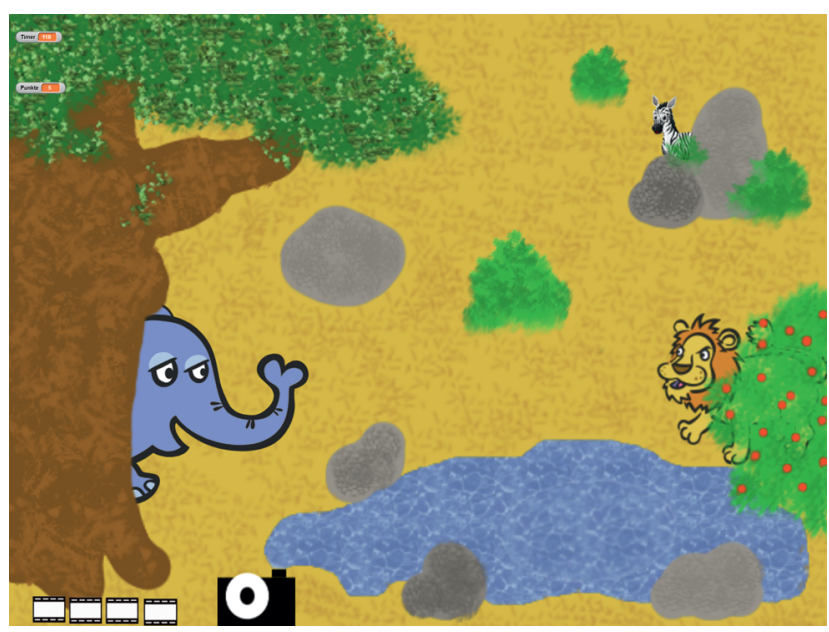

Abb. 2: Screenshot einer studentischen Arbeit. In Snap! sollte ein Schießspiel programmiert werden. Die Studierenden implementierten eine Fotosafari.

In der zweiten Phase des Moduls erfolgt daher der Umstieg auf eine „richtige“ Programmiersprache, in diesem Fall Python. ${ }^{5}$ Python ist eine quelloffene, für alle Betriebssysteme verfügbare Programmiersprache, die eine einfache Syntax und eine Vielzahl an sehr gut dokumentierten Funktionsbibliotheken für alle denkbaren Zwecke und Szenarien bereitstellt. Mit PyCharm Edu ${ }^{6}$ steht darüber hinaus eine kostenlose integrierte Entwicklungsumgebung zur Verfügung, die den Einstieg in erste Programmierprojekte durch einen sehr guten Editor mit Autovervollständi-

5 https://www.python.org.

6 https://www.jetbrains.com/pycharm-edu. gung, Verknüpfung mit der Python-Dokumentation und Syntax-Hervorhebung erleichtert. Die Studierenden erkennen die bereits in Snap! vermittelten Konzepte wie Variablen, Bedingungen, Schleifen, einfache Datenstrukturen und Funktionen in Python wieder und können so fließend von Snap! zu Python wechseln. Bei Bedarf können verschachtelte Abläufe zunächst in Snap! entworfen und nachvollzogen werden und dann nach Python übertragen werden. Auch in dieser Phase werden die Studierenden aufgefordert, sich neben den Materialien der Vorlesung weitere Lernmaterialien zu suchen, für sich auszuprobieren und sie der Semestergruppe vorzustellen. Das Modul schließt mit einem Gruppenprojekt, in dem ein einfaches Spiel in Python erarbeitet wird.

\subsection{Informationssysteme 2: Daten}

Das zweite Modul stellt die Arbeit mit Daten in den Mittelpunkt. Die Aufgaben bauen hierbei stets auf „realen“ Beispieldaten aus öffentlich verfügbaren Quellen auf und behandeln typische, immer wiederkehrende Problemstellungen wie das Speichern und Laden dieser Daten, die Abbildung in Datenstrukturen innerhalb des Programms und das Bearbeiten, Filtern, Verarbeiten und Suchen einzelner Datensätze. Auf der technischen Seite werden dabei die Python-Standardbibliotheken vorgestellt, die für alle gängigen Dateiformate und Datenbankanbindungen existieren und somit vom konkreten Format oder Datenbankprodukt abstrahieren. Da diese Bibliotheken alle ähnlich aufgebaut sind, kann ein Programm, das mit lokalen Dateien arbeitet, mit wenigen Änderungen an einigen Funktionsaufrufen auf ein anderes Dateiformat oder gar eine Datenbankanbindung umgestellt werden. Dies erlaubt eine 
kontinuierliche Weiterentwicklung der Programme in den Aufgaben, die während des Semester zu bearbeiten sind: Werden zunächst tabellenartig strukturierte Daten aus CSV-Dateien gelesen und verarbeitet, folgen komplexere Datenstrukturen, die als JSON-Daten verarbeitet werden können. Sobald die Größe der Daten ein lineares Durchsuchen zu aufwändig erscheinen lässt, kann die Speicherung und Indexierung in einem Datenbanksystem erfolgen. Hier werden zunächst die für komplexe Daten wesentlich einfacher einsetzbaren dokumentorientierten noSQL-Datenbanken vorgestellt, um die internen Datenstrukturen möglichst unverändert in der Datenbank wiederzufinden.

Relationale Datenbanken und auch das XML-Format werden dabei natürlich nicht ausgespart, sondern als wichtige „Legacy“-Elemente der Datenverarbeitung in eigenen Aufgaben vorgestellt, ebenso wie die Funktionsbibliotheken zu deren Verarbeitung.

Als Prüfungsleistung werden in Gruppen zwei größere Programmierprojekte bearbeitet, um die bis zu diesem Zeitpunkt erlernten Techniken aktiv und eigenständig zum Einsatz zu bringen. Beispielsweise kann dies eine Selektion von Daten aus einem größeren Datensatz des Statistischen Bundesamtes oder die Aggregation von Daten aus der Kriminalitätsstatistik des Bundeskriminalamts (BKA) sein. In Einzelvorträge stellen die Studierenden darüber hinaus ihren Kommilitoninnen und Kommilitonen ein konkretes Datenformat, seine Geschichte und Anwendung sowie die Verarbeitung mittels Python-Bibliotheken vor.

\subsection{Informationssysteme 3 : Web}

Das dritte Modul startet mit einer Einführung in den Aufbau des http-Protokolls, in Webserver und in die Webstandards HTML5 und CSS. Dabei wird der Webserver ausgehend von den formalen Definitionen sukzessive gemeinsam programmiert und um zusätzliche Funktionen erweitert, z.B. das Ausführen von in Python-Dateien gespeicherten separaten Skripten. In der zweiten Hälfte folgt ein umfangreiches Gruppenprojekt. Die Studierenden müssen eine eigene Idee für eine Webseite entwickeln und diese systematisch realisieren. Inhalte müssen definiert und strukturiert werden; die Studierenden müssen eine angemessene grafische Gestaltung finden und umsetzen sowie interaktive Elemente programmieren. Dabei kommt der gemeinsam entwickelte Webserver zum Einsatz, der von den Studierenden für die Umsetzung ihrer Ideen auch verändert und angepasst werden kann. Es gibt keine strengen Vorgaben zu den Themen und Ideen der Webseite, die Studierenden haben hier große Freiheiten und sind auch in der Aufgabenteilung innerhalb der Gruppe frei. Wichtig ist nicht nur das Ergebnis, sondern auch der Prozess von der Idee über eine Anforderungsanalyse und ein Grobkonzept bis zur Umsetzung ist umfassend zu dokumentieren und der individuelle Beitrag der einzelnen Studierenden $\mathrm{zu}$ kennzeichnen. Abb. 3 und 4 zeigen Screenshots von Beispielarbeiten.

\subsection{Evaluation der neuen Module}

Zum Ende des Wintersemesters 2018/19 haben drei Kohorten alle drei Module vollständig durchlaufen und somit stehen ausreichend Erfahrungswerte von Seiten der Lehrenden und Rückmeldungen der Studierenden im Rahmen der hochschulweiten Vorlesungsevaluation bereit. Die wichtigsten Erkenntnisse sind:

- Die Bedeutung von IT-Kompetenzen für das Berufsfeld wird von einem Teil der Studierenden falsch eingeschätzt. Beispielsweise wird öfter angenommen, dass diese in öffentlichen Bibliotheken kaum benötigt werden. In Gesprächen mit solchen Studierenden zeigt sich, dass hierbei Wunschvorstellungen bezüglich der eigenen künftigen Tätigkeit eine Rolle spielen und nur unvollständiges Wissen über die Bedürfnisse der Berufspraxis vorhanden ist.

- Der Anspruch, auch Studierende mit geringen oder gar keinen Vorkenntnissen und eher skeptischer Haltung zur Thematik erreichen zu wollen, wird erfüllt. Mehrere Studierende haben in der Evaluation oder auch im persönlichen Gespräch sehr deutlich formuliert, dass sie sich zu Beginn des Studiums nicht zugetraut hätten, die Module erfolgreich zu bearbeiten - nun aber sogar Vertiefungen in diesem Bereich belegen möchten.

Eine Auswertung der Noten hat außerdem ergeben, dass diejenigen Studierenden, die die IT-Module nicht erfolgreich abgeschlossen haben, auch in den anderen fachlichen Modulen teilweise massive Defizite aufweisen. Zum derzeitigen Zeitpunkt hat niemand ausschließlich wegen der Module der Säule Informationssysteme das Studium abgebrochen. Vielmehr zeigt sich, dass Studierende, die sich auf das Lehrkonzept des „flipped classroom“ eingelassen, regelmäßig die Aufgaben bearbeitet und sich aktiv am Unterricht beteiligt haben, mit überdurchschnittlichen Noten abgeschlossen haben.

- Die gewünschten (über)fachlichen Kompetenzen werden erreicht. Die abgegebenen Programme funktionieren in der Regel wie gefordert und ihre Struktur ist nachvollziehbar. Zwar finden sich oft umständliche oder wenig optimierte Lösungen, doch ist eine perfek- 


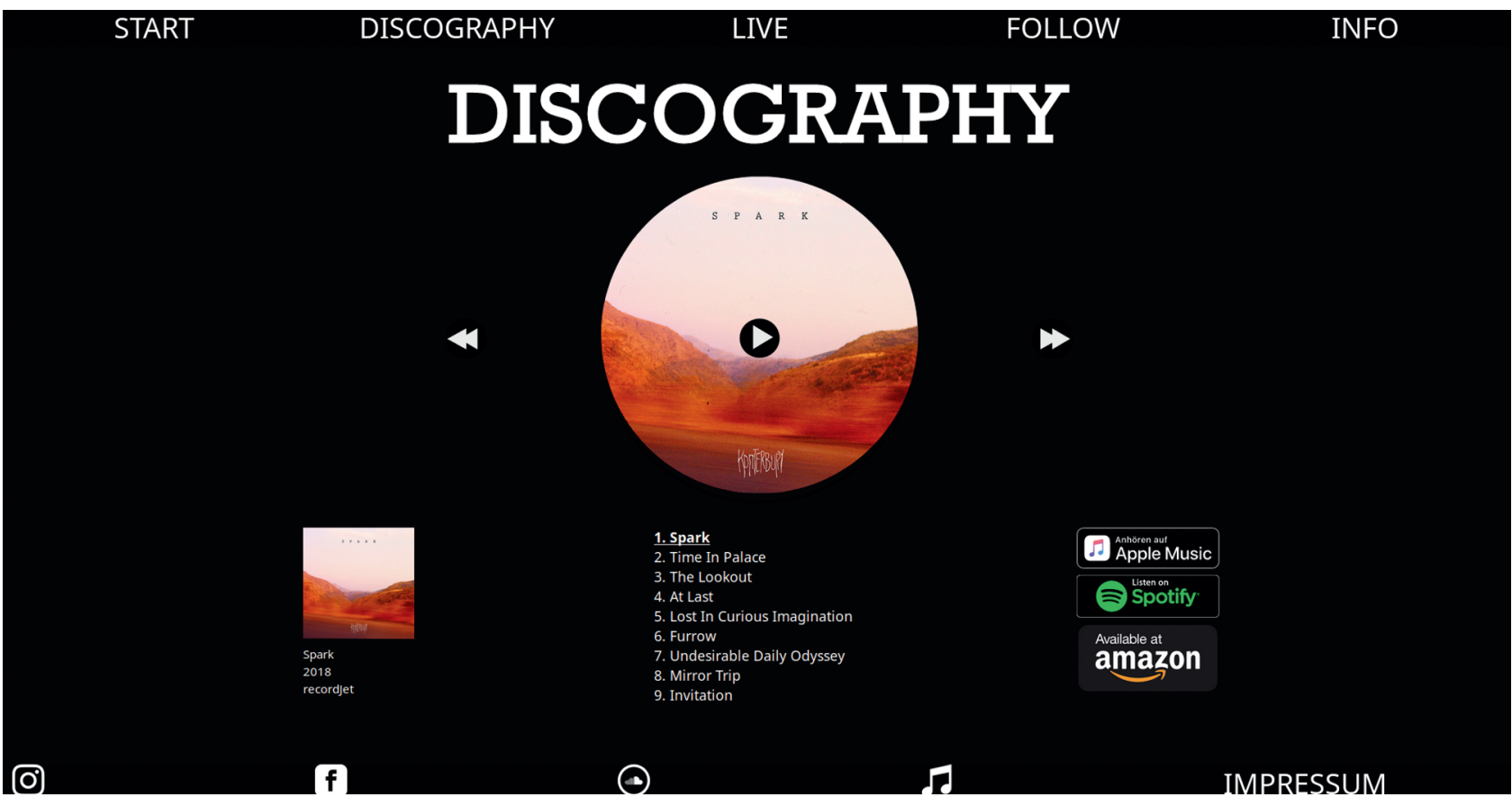

Abb. 3: Screenshot einer studentischen Arbeit aus dem Bereich Web: Eine Seite für eine real existierende Band, mit der Möglichkeit, Alben und Songs der Band auf der Seite direkt zu hören.

\section{gartner in Crime}

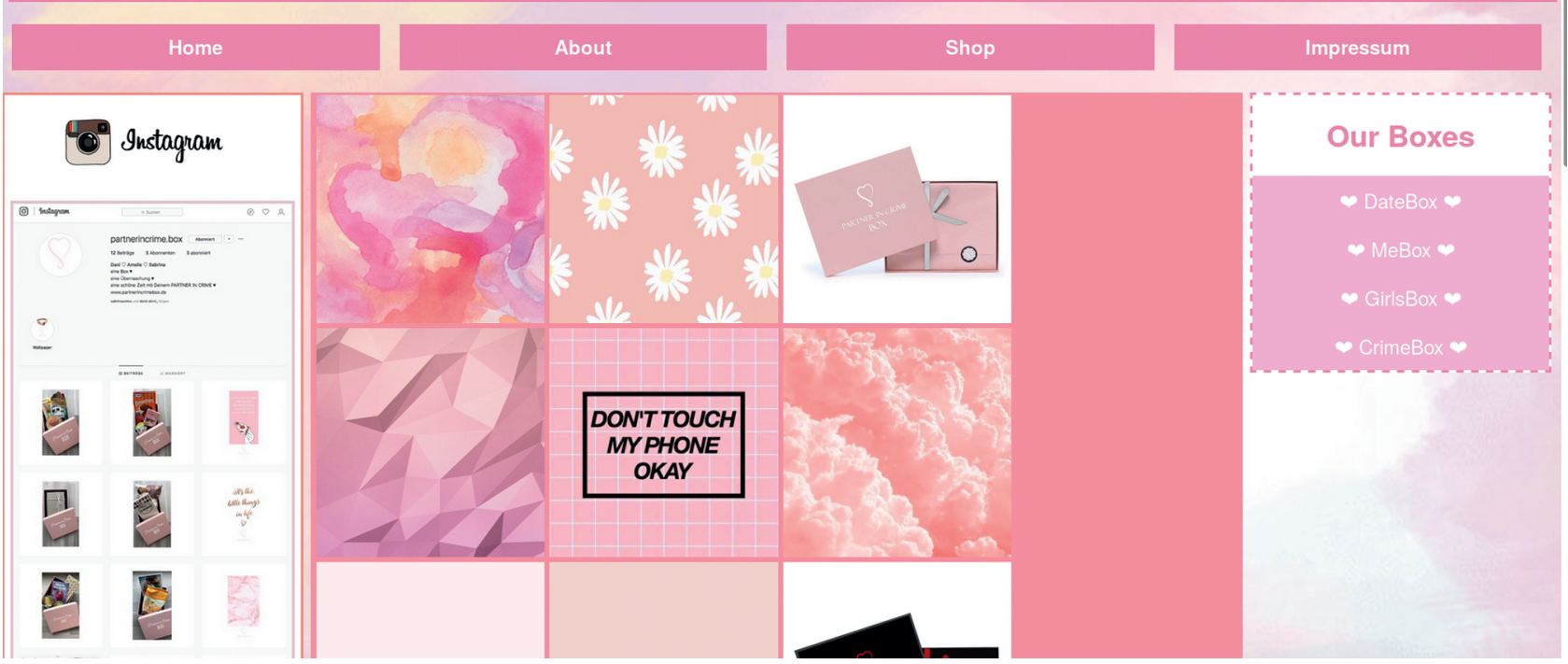

Abb. 4: Screenshot einer studentischen Arbeit aus dem Bereich Web: Die Seite eines fiktiven Online-Shops, über den individuell gestaltete „Event-Boxen“verkauft werden.

te Programmierung auch gar nicht das Ziel der Veranstaltung - wenn das Problem gelöst ist, ist die Aufgabe erfolgreich bearbeitet. Gerade das freie Projekt im dritten Semester überrascht außerdem mit sehr umfangreichen und gut umgesetzten Ideen. Abb. 3 zeigt zwei Beispiele studentischer Arbeiten.
- In den mittlerweile stattfindenden Vertiefungsfächern im Hauptstudium, wie z. B. Daten- und Textanalyse, treffen die Studierenden auf Teilnehmer aus anderen Studiengängen, insbesondere der Wirtschaftsinformatik. Es lässt sich feststellen, dass es im Hinblick auf die Anforderungen dieser Veranstaltun- 
gen keinen nennenswerten Unterschied in den Programmierfähigkeiten der Studierenden gibt. Durch die Umstrukturierungen wurden derartige Vertiefungen mit einem Fokus auf praktische Erfahrungen mit der Umsetzung von Analysen mittels eigener Programme erst möglich. Sie werden von den Studierenden als Angebot, die eigenen Programmierfähigkeiten weiter zu entwickeln, gut angenommen.

\section{Schwerpunkt „Daten- und Informationsmanagement (DIM)،}

Die im Jahr 2011 angestoßene Auseinandersetzung mit den Inhalten und dem didaktischen Rahmen des Studiums wurde auch nach der fakuktätsweiten Umstellung weitergeführt.

Eine im Rahmen des internen Qualitätsmanagement durchgeführte Reakkreditierung war der Anlass, die Erfahrungen mit dem neuen Modell zu reflektieren und mit einer angepassten Studienordnung in den Begutachtungsprozess zu gehen. Die Grundidee dafür war, die Inhalte und Wahlmöglichkeiten stärker vorzustrukturieren und dies durch die Ausweisung von zwei unterschiedlichen Schwerpunkten zu verdeutlichen. So sollen sowohl die unterschiedlichen Anforderungen aus der Berufspraxis besser abgebildet werden als auch potentiellen Studierenden die Inhalte und Wahlmöglichkeiten des Studiums besser kommuniziert werden.

Unter dem neuen Namen „Informationswissenschaften“ wird einer der beiden Schwerpunkte Bibliotheks-, Kultur- und Bildungsmanagement (BKBM) und Daten- und Informationsmanagement (DIM) nach einem gemeinsamen Grundstudium zum dritten Semester gewählt. An das Hauptstudium mit sowohl gemeinsamen als auch schwerpunktspezifischen Pflichtmodulen schließt sich nach dem praktischen Studiensemester unverändert das fakultätsweite Projektstudium an. Auch die Angebote zu den Schlüsselkompetenzen bleiben inhaltlich unverändert. Abb. 5 zeigt die Module in der Übersicht. ${ }^{7}$

In beiden Schwerpunkten sind wieder 15 ECTS für allgemeine IT-Kompetenzen eingeplant. Neben der Einführung in das Programmieren sind nun im ersten Semester erweiterte Grundlagen IT-basierter Systeme vorgesehen. Damit soll der Beobachtung Rechnung getragen werden, dass die Studierenden sehr unterschiedliche Vorkenntnis-

$7 \mathrm{Zu}$ den Details der Studienordnung und dem Prozess zur Entwicklung der einzelnen Module siehe Wiesenmüller (2018). se mitbringen und Kompetenzen in der Bedienung von Standardsoftware und der Medienerstellung und -gestaltung, die vor wenigen Jahren noch selbstverständlich waren, nicht mehr in der Breite vorhanden sind. Im zweiten Semester folgt das Modul Web-Technologien, das die Inhalte des zuvor angebotenen Web-Moduls aufgreift.

Im Schwerpunkt „Daten- und Informationsmanagement“ folgen im dritten Semester im Modul „Daten und Datenintegration“ die Inhalte des bisherigen Daten-Moduls. Als 10-ECTS-Modul konzipiert, steht mehr Zeit für Übungen zur Verfügung, in denen eine größere Vielfalt an Datenformaten, Datenquellen und Datenstrukturen vermittelt werden kann. Ein weiterer Aspekt ist die Anreicherung von Daten über Web-APIs und die Zusammenführung von Daten aus unterschiedlichen Quellen. Im parallel stattfindenden Modul „Web-Programmierung“ steht die serverseitige Programmierung von Web-Anwendungen im Mittelpunkt. Ausgehend von einfachen Beispielen wie Datenbanken, die über ein Webinterface abgefragt und ergänzt werden können, wird der Aufbau webbasierter Systeme erläutert und die Gruppen erstellen komplexere Anwendungen. Im vierten Semester folgt das komplett neu konzipierte Modul „Metadatenmanagement“, in dem verschiedene Standards, Bewertung und Einordnung von Metadaten, typische Arbeitsabläufe und anwendungsspezifische Werkzeuge, primär im Kontext beschreibender Daten, Thema sind. Auch hier wird neben dem Faktenwissen durch praktische Übungen sichergestellt, dass die Studierenden typische Aufgabenstellungen selbstständig bearbeiten können.

Im Wahlpflichtbereich können verschiedene Aspekte des Informationsmanagements weiter vertieft werden. Die Angebote umfassen

- Forschungsnahe Dienstleistungen,

- Daten- und Textanalyse,

- Open Government und Open Data,

- Datenbanken,

- Information Retrieval,

- IT-Management,

- Software-Entwicklung,

und decken somit ein sehr breites Spektrum ab. Im Rahmen des Projektstudiums werden darüber hinaus regelmäßig fachspezifische Projekte aus diesen Bereichen mit Partnern aus der Praxis angeboten.

Die Erfahrungen mit den neuen IT-Grundlagenmodulen und den ersten erfolgreich abgeschlossenen Wahlveranstaltungen und Projekten sind durchwegs positiv. Sie zeigen, dass es nicht nur möglich ist, einen niederschwelligen, motivierenden Zugang zu informationstechnischen Grundlagen anzubieten, sondern darauf aufbauend immer 


\begin{tabular}{|c|c|c|c|c|c|c|}
\hline 1 & Märkte und Kunden & $\begin{array}{c}\text { Datenstrukturierung } \\
\text { und Recherche }\end{array}$ & \multicolumn{2}{|c|}{$\begin{array}{l}\text { IT-Grundlagen und Einführung ins } \\
\text { Programmieren }\end{array}$} & $\begin{array}{l}\text { Wissenschaftliche } \\
\text { Grundlagen } 1\end{array}$ & $\begin{array}{l}\text { Orientierung und } \\
\text { Kurzpraktikum }\end{array}$ \\
\hline 2 & $\begin{array}{l}\text { Angebots- und } \\
\text { Dienstleistungs- } \\
\text { entwicklung }\end{array}$ & Medienerschließung & Web-Technologien & $\begin{array}{l}\text { Lernen und } \\
\text { Lehren }\end{array}$ & $\begin{array}{l}\text { Wissenschaftliche } \\
\text { Grundlagen } 2\end{array}$ & Ways of Working \\
\hline \multicolumn{7}{|c|}{ Schwerpunkt Bibliotheks-, Kultur- und Bildungsmanagement } \\
\hline 3 & $\begin{array}{c}\text { Informationssysteme } \\
\text { und } \\
\text { Geschäftsprozesse }\end{array}$ & $\begin{array}{l}\text { Cultural Heritage, } \\
\text { Digitalisierung }\end{array}$ & \multicolumn{2}{|c|}{$\begin{array}{l}\text { Public Management und } \\
\text { Kulturmanagement }\end{array}$} & $\begin{array}{l}\text { Gesellschaftliche und } \\
\text { kulturelle Kontexte }\end{array}$ & Tools for Working \\
\hline 4 & Open Society & $\begin{array}{l}\text { Architektur und } \\
\text { Einrichtung }\end{array}$ & Wahlpflicht & Wahlpflicht & Wahlpflicht & $\begin{array}{c}\text { Working in a Media } \\
\text { World }\end{array}$ \\
\hline \multicolumn{7}{|c|}{ Schwerpunkt Daten- und Informationsmanagement } \\
\hline 3 & $\begin{array}{c}\text { Informationssysteme } \\
\text { und } \\
\text { Geschäftsprozesse }\end{array}$ & $\begin{array}{l}\text { Cultural Heritage, } \\
\text { Digitalisierung }\end{array}$ & \multicolumn{2}{|c|}{ Daten und Datenintegration } & Web-Programmierung & Tools for Working \\
\hline 4 & Open Society & $\begin{array}{l}\text { Metadaten- } \\
\text { management }\end{array}$ & Wahlpflicht & Wahlpflicht & Wahlpflicht & $\begin{array}{l}\text { Working in a Media } \\
\text { World }\end{array}$ \\
\hline 5 & \multicolumn{6}{|c|}{ Praktisches Studiensemester } \\
\hline \multirow{2}{*}{6} & \multirow{2}{*}{\multicolumn{5}{|c|}{ Interdiziplinäres Projektstudium }} & Wahlpflicht \\
\hline & & & & & & Wahlpflicht \\
\hline \multirow{2}{*}{7} & \multirow{2}{*}{\multicolumn{2}{|c|}{ Interdisziplinäres Projektstudium }} & Wahlpflicht & \multirow{2}{*}{\multicolumn{3}{|c|}{ Bachelorarbeit und Kolloqium }} \\
\hline & & & Wahlpflicht & & & \\
\hline
\end{tabular}

Abb. 5: Studienverlauf mit Modulbezeichnungen der seit dem Wintersemester 2018/19 gültigen Studienordnung

komplexere Inhalte auf einem technisch hohen Niveau zu vermitteln. Die Anforderungen der Berufspraxis und die neuen Tätigkeitsfelder sind aus unserer Sicht zurzeit einem starken Wandel unterzogen. Somit kann die Fähigkeit, sich eigenständig und zielgerichtet in neue Technologien, Programmiersprachen oder Abläufe einzuarbeiten, kaum hoch genug eingeschätzt werden. Durch die Breite der inhaltlichen Angebote und die zahlreichen Möglichkeiten, das Gelernte zeitnah praktisch umzusetzen, sind wir zuversichtlich, dass die Studierenden des Schwerpunkts „Daten- und Informationsmanagement“ alle Voraussetzungen für die künftigen Tätigkeitsfelder im Kontext von Informationseinrichtungen und Daten mitbringen, ob als Data Librarian, Research Data Manager oder Metadata Expert.

\section{Literaturverzeichnis}

Simon, Ingeborg; Vonhof, Cornelia (2008): Bibliotheks-und Informationsmanagement: Bachelor-und Master-Abschlüsse in Stuttgart. In: AKMB-news: Informationen zu Kunst, Museum und Bibliothek, 14 (2), 10-14.
Vonhof, Cornelia (2017): Bachelor und Berufspraxis-das Studienmodell der Hochschule der Medien Stuttgart. In: Bibliotheksdienst, 51 (10-11), 923-34.

Wiesenmüller, Heidrun (2018): Nach der Studienreform ist vor der Studienreform - dynamische Weiterentwicklung an der Hochschule der Medien. In: Südwest-Info Nr. 31, hrsg. vom Regionalverband Südwest des Vereins Deutscher Bibliothekarinnen und Bibliothekare e.V. (VDB). Verfügbar unter https://www.vdb-on line.org/landesverbaende/sw/sw-info/suedwest-info-31-2018. pdf.

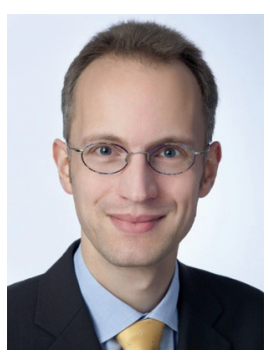

Magnus Pfeffer

Hochschule der Medien

Fakultät Information und Kommunikation

Nobelstr. 10

70569 Stuttgart

pfeffer@hdm-stuttgart.de 